\title{
NÚCLEO DE AÇÕES DISCENTES DA ESCOLA DE ENGENHARIA DA UNIVERSIDADE FEDERAL DO RIO GRANDE DO SUL: PROJETO ACOLHIMENTO DOS CALOUROS DA ESCOLA DE ENGENHARIA
}

DOI: 10.37702/2175-957X.COBENGE.2021.3544

Giovanni Falcão Mendes - giovannimendes800@gmail.com

Universidade Federal do Rio Grande do Sul

Rua Duque de Caxias, 15761576

90010-281 - Porto Alegre - RS

Simone Ramires - simone.ramires@ufrgs.br

Universidade Federal do Rio Grande do Sul

Praça Argentina 9

90040-020 - Porto Alegre - RS

Helena Beatriz Petersen - helena.petersen@ufrgs.br

Universidade Federal do Rio Grande do Sul

Rua Castro Alves 549

90430-131 - Porto Alegre - RS

Resumo: O Núcleo de Ações Discentes da Escola de Engenharia - NADI/EE da Universidade Federal do Rio Grande do Sul (UFRGS) tem a finalidade de apoiar os discentes no desenvolvimento de suas atividades. Assim, buscando auxiliá-los dentro das competências propostas, permanência, integração e participação no universo acadêmico. Não obstante, com que se sintam parte fundamental da Universidade, num contexto de participação ativa durante a sua trajetória acadêmica. Sendo assim, surge o projeto Acolhimento dos Calouros da EE - Desafio "Como tornar a UFRGS mais sustentável" que tem como objetivo possibilitar aos calouros dos 13 cursos da EE, identificar lacunas acerca dos problemas encontrados na vivência da Universidade, por exemplo, gestão de resíduos sólidos, espaços de convivência, revitalização de áreas, energias renováveis. Na edição de 2020/1, foram apresentados 7(sete) projetos, no qual 4 (quatro) são destaques, entre eles: Reliqua Biogás, Naturae Plastic, GURI: rotas interativas, CONSERTA UFRGS. E, a partir desse projeto, é possível desenvolver experiências vivenciais, no calouro, que permitam a construção de diversos conhecimentos. Esses que fomentam a inovação, a criatividade e o espírito empreendedor dos discentes de 


\section{COBENGE

diferentes Engenharias da UFRGS. Do mesmo modo, suscitar o debate acerca das etapas para o empreendimento de um negócio, analisar os elementos influenciadores neste processo e discutir questões de posicionamento em mercado, por exemplo, verificar o investimento inicial para iniciar o projeto e o retorno(payback) destes.

Palavras-chave: Engenharia. Sustentabilidade. Inovação. Desafio. 


\section{(c) COBENGE \\ 28 a 30 de SETEMBRO \\ NÚCLEO DE AÇÕES DISCENTES DA ESCOLA DE ENGENHARIA DA UNIVERSIDADE FEDERAL DO RIO GRANDE DO SUL: PROJETO ACOLHIMENTO DOS CALOUROS DA ESCOLA DE ENGENHARIA}

\section{INTRODUÇÃO}

A percepção da falta de engenheiros qualificados no país aumenta quando a Indústria requer profissionais que ultrapassem os limites do pensamento objetivo e quantitativo tradicional. Infere-se que é necessário a melhoria nas competências interpessoais, na educação primária e no estímulo da formação técnica e científica, além de demandar mais das habilidades cognitivas do engenheiro, as que se referem a inovação, como a criatividade, criticidade, indução, empreendedorismo, análise de riscos e ser capaz de resolver problemas (CONFEDERAÇÃO NACIONAL DA INDÚSTRIA, 2015).

Sendo assim, as análises acadêmicas, da educação empreendedora na formação de engenheiros, abrangem as propostas, modelos e métodos de aprendizagem de empreendedorismo (MÄKIMURTO-KOIVUMAAA \& BELT, 2015), papéis e importância do empreendedorismo (BYERS et al, 2013), impactos do empreendedorismo (JARRAR \& ANIS, 2016), instrumentos de avaliação de empreendedorismo em engenharia (BAILEY \& SZABO, 2006) e novas habilidades empreendedoras do engenheiro no século XXI (ABDULWAHED \& HASNA, 2017).

Portanto, o projeto tem a finalidade de auxiliar no planejamento, intervir com metodologias que propiciem ao acadêmico observar, idealizar, prototipar e unir diversas perspectivas de problema, priorizando o trabalho colaborativo e multidisciplinar. Além disso, possibilitar ao acadêmico: visão, liderança, energia, saber ouvir e argumentar, ampliação do networking, capacidade de resolução de problemas, saber trabalhar em equipe e domínio de outros idiomas (FILION, 1993; GIBB, 2002; CNI, 2006, 2010, 2015).

A partir disso, a Universidade Federal do Rio Grande do Sul compreendeu seu papel como peça-chave e teve a percepção de que o desafio de formar engenheiros empreendedores e inovadores surge desde o momento em que os calouros são inseridos na Universidade. Para isso, implementou o projeto "DESAFIO DOS CALOUROS DOS CALOUROS DA ESCOLA DE ENGENHARIA - Como tornar a UFRGS mais sustentável" realizado pelo Núcleo de Ações Discentes - NADI em parceria com o Instituto de Física e Instituto de Pesquisas Hidráulicas - IPH para que os calouros sejam desafiados a propor projetos de melhorias, que tenham viés sustentável, abrangendo as esferas econômica, social e ambiental e ainda alinhar com os 17 Objetivos do Desenvolvimento Sustentável da ONU.

Como resultado dessa proposta foi possível observar o surgimento de vários projetos na edição 2020/1 relacionados à sustentabilidade e melhoria das condições de infraestrutura da Universidade, entre eles, GURI: rotas interativas, CONSERTA UFRGS, Reliqua Biogás, Naturae Plastic (plástico biodegradável).

Nesse sentido, o presente estudo tem o objetivo de apresentar e discutir conceitos como o engajamento do curso de engenharia com a sociedade, a necessidade e a importância do acolhimento de calouros, a identificação de lacunas como habilidades e competências de um engenheiro, inovação e empreendedorismo, sustentabilidade e responsabilidade social, assim como apresentar a proposta dos projetos destaques. 


\section{NECESSIDADE E IMPORTÂNCIA DE ACOLHIMENTO DOS CALOUROS}

Frequentemente ocorre de estudantes recém-chegados à universidade (calouros), sentem-se deslocados, visto que muitas vezes vêm de outras cidades, estados ou até mesmo de diferentes países, quando entram na universidade não possuem amigos próximos ou sofrem com a dificuldade de integração. Entretanto, atualmente muitas Universidades, como por exemplo a Universidade Federal do Rio Grande do Sul (UFRGS), aderiram a criação de campanhas e desafios para os calouros para que dessa forma consigam entender o funcionamento da universidade, e ajudá-los a se agrupar e interagir com outros cursos e estudantes.

Usando essa estratégia, além do que propõe as Novas Diretrizes Curriculares dos Cursos de Graduação em Engenharia, Resolução ํㅡ 2 de 29 de abril de 2019 que deve estabelecer programa de acolhimento para os calouros, faz com que aproveitem melhor as oportunidades que o curso e a Universidade pode prover, tanto para sua vida profissional, tanto para o desenvolvimento psicológico.

Visto que, normalmente, a maior parte dos calouros vêm direto do Ensino Médio Regular, onde tem seus grupos de amizades e onde passam a maior parte do dia. E que, quando mudam para outro ambiente é bem comum se sentirem deslocados, principalmente nos primeiros semestres do curso. Por isso com a ajuda e incentivo da Universidade muitos conseguem crescer e desenvolver um pensamento mais maduro diante dos desafios, conseguem superar alguns de seus medos. Com tudo isso é possível observar a importância das atividades de acolhimento que muitas Universidades estão aderindo atualmente.

\section{IDENTIFICAÇÃO DE LACUNAS}

A UFRGS integra milhares de alunos, professores, pesquisadores e funcionários, inevitavelmente tem problemas e falhas estruturais nas áreas de serviços, processos e produtos. Com a visão e pensamento fora da caixa por parte dos alunos que não conhecem a UFRGS é possível a identificação das lacunas que passariam despercebidas para outras pessoas que convivem com o problema e se acostumaram. Tendo isso em mente, a escola de engenharia desenvolveu um projeto a fim de preencher essa necessidade dos alunos de integração e adaptação em um ano tão crítico com a da universidade de se modernizar e se tornar cada vez mais sustentável. É proposto aos alunos que, por meio de suas experiências e o uso de metodologias de análise de falhas, criem projetos que tenham um viés que se alinhe aos objetivos de desenvolvimento sustentável da ONU.

Algumas dessas metodologias já são conhecidas e vastamente utilizadas na Engenharia, como Cinco Porquês e Diagrama de Ishikawa (também conhecido como "Espinha de Peixe") (ENGETELES, 2019). Essas ferramentas ajudam a aprimorar uma das competências essenciais de um engenheiro, a identificação de lacunas. Sendo assim, os alunos da Universidade são introduzidos à rotina universitária: pesquisa; análise de dados; comunicação com diversas pessoas e entidades; e consequentemente, integração e união entre os calouros e a instituição de ensino.

\section{$4 \quad$ SUSTENTABILIDADE E RESPONSABILIDADE SOCIAL}

Ações são caracterizadas como sustentáveis quando visam suprir as necessidades atuais dos seres humanos sem comprometer o futuro da sociedade, ou seja, ela está diretamente relacionada ao desenvolvimento econômico e material quando não há agressão ao meio ambiente, usando os recursos naturais de forma inteligente para que eles 
não se esgotem posteriormente. Concomitantemente, segundo pereira (2017) a responsabilidade social é a responsabilidade que uma instituição toma em prol da sociedade, com o objetivo de melhorar a qualidade de vida da sociedade e cuidar do meio ambiente.

Partindo-se do pressuposto que tecnologias do futuro irão impulsionar negócios mais sustentáveis, abrindo um leque de opções para as empresas agregarem valor, torna-se essencial descobrir novas formas para empresas, que atuam em ambientes cada vez mais complexos, incorporarem a visão de sustentabilidade econômica, social e ambiental em suas estratégias de negócios. Isto porque as tecnologias que impulsionam negócios bemsucedidos e sustentáveis são aquelas que, desde a formulação estratégica até as etapas finais do processo de pesquisa e desenvolvimento ( $p \& d)$ e de inovação tecnológica incorpora os valores éticos da sustentabilidade (Corrêa, 2010).

\section{$5 \quad$ PROJETOS DESTAQUES}

\subsection{Reliqua Biogás}

Reliqua Biogás é proposto por calouros da Escola de Engenharia da Universidade Federal do Rio Grande do Sul no projeto Acolhimento dos Calouros da EE - Desafio "Como tornar a UFRGS mais sustentável". É composto por seis integrantes, dos cursos de Engenharia Física, Engenharia de Materiais, Engenharia Mecânica e Engenharia Química.

O projeto em questão estuda a viabilidade da implementação de um biodigestor para geração de biogás no Hospital de Clínicas de Porto Alegre - HCPA e é alicerçado nos 17 Objetivos do Desenvolvimento Sustentável - ODS da Organização das Nações Unidas, entre eles, o objetivo 7 de assegurar o acesso confiável, sustentável, moderno e a preço acessível à energia para todos, o objetivo 11 de tornar as cidades e os assentamentos humanos sustentáveis e o objetivo 13 de tomar medidas para combater a mudança climática e seus impactos (ONU, 2015).

Neste sentido, o projeto justifica-se pela necessidade de redução de custos quanto a geração de resíduos sólidos orgânicos do Hospital de Clínicas de Porto Alegre (HCPA), pela geração de Biofertilizante, biogás para geração de energia térmica, em substituição ao GLP, gasodutos GN e/ou energia veicular como GNV há uma considerada redução no potencial de poluição do meio ambiente, uma vez que é composto por uma alta concentração de gás metano $\left(\mathrm{CH}_{4}\right)$, cerca de 24 vezes superior ao dióxido de carbono $\left(\mathrm{CO}_{2}\right)$, no que se refere gases de efeito estufa (GEE).

\subsection{GURI: Rotas alternativas}

Projeto guri representa um aplicativo para smartphone e mapas interativos que têm a função de apresentar caminhos aos usuários de forma que consigam se guiar no interior dos campi, diferenciando os prédios por cores e trazendo rotas interativas a partir desses prédios, com o uso de placas e QR code, que ao ser escaneado apresenta uma rota ao destino desejado. Considerando que muitas vezes a localização das salas de aula dentro dos prédios também é de difícil acesso, foi expandido o foco principal do projeto para ajudar na localização das salas, a partir de representações do interior dos prédios. Essas representações serão feitas a partir da consulta de documentos atualizados da universidade e de visitas às salas realizadas pelos próprios participantes. Ou seja, mapas externos (localizados nos campi) com QR code guiarão os usuários aos prédios, e mapas internos (localizados no interior dos prédios) com QR code guiarão os usuários às salas. No aplicativo, além de existir a opção de escanear qualquer QR code para gerar alguma rota, 
também será possível consultar os mapas externos e internos para conhecer os campis e prédios.

\subsection{Conserta UFRGS}

O projeto Conserta UFRGS tem como finalidade a criação de um aplicativo que aproxima estudantes, funcionários e professores para auxiliarem na otimização do serviço de manutenção da universidade. Através dele é criada uma rede de colaboração e instalada uma cultura do cuidado dentro da instituição, facilitando e agilizando desde a identificação das avarias estruturais do ambiente universitário até a conclusão da manutenção de tais.

Com o intuito de reduzir o desperdício de água, luz e entre outros, o projeto se preocupa também com a parte financeira. Sabe-se que a universidade não detém grandes recursos financeiros para a realização da manutenção, portanto o aplicativo propõe a possibilidade ao reclamante de doar voluntariamente certa quantia de valor para a realização do reparo. Esse montante seria captado por meio da compra de tickets de manutenção previamente pagos que se assemelhariam aos atuais tickets do RU (Restaurante Universitário) que são ofertados aos estudantes e professores. Outra maneira de auxiliar financeiramente as manutenções na universidade, seria a partir do Fundo Centenário. Esse, já existente, poderia receber doações de maiores quantias internas e externas de, por exemplo, ex-alunos por meio de pessoa física e/ou jurídica.

O aplicativo seria criado por empresas parceiras especializadas e na tela inicial haverá a seleção de categorias que necessitam de manutenção, entre elas:

a. $\quad$ Elétrica: computadores, projetores, ar-condicionado, luzes, tomadas etc.;

b. Sanitária: entupimento; vazamento; quebra de vaso sanitário, porta, pia, saboneteira, porta papel toalha etc.;

c. Materiais: classes, cadeiras, quadros, piso, teto, paredes etc.

Dessa forma, a infraestrutura da universidade se manteria mais conservada e apresentaria maior qualidade para seus alunos, professores e funcionários.

\subsection{Naturae Plastic}

O projeto Naturae Plastic surge para reduzir o consumo de plásticos descartáveis na universidade federal do rio grande do Sul, utilizando a infraestrutura da instituição em conjunto com o laboratório de polímeros. O projeto possui como objetivo principal avaliar novas fontes de matéria prima mais sustentáveis para a construção de copos descartáveis, possuindo como norte a análise de biopolímeros oriundos da biomassa residual de arroz, valorizando a economia circular da sua cadeia produtora.

Busca-se com esse projeto desenvolver um aproveitamento sustentável da biomassa residual da indústria do arroz para a produção de copos e embalagens plásticas e biodegradáveis de origem vegetal como alternativa para substituição dos não biodegradáveis e de origem fóssil (polipropileno e poliestireno), contribuindo para a redução desses resíduos, além de incrementar a economia circular desse grão, agregando valor à sua indústria.

Pretende-se amenizar os efeitos negativos relacionados ao meio ambiente causados pelos descartáveis, ao mesmo tempo que cria uma proposta empreendedora, unindo inovação e sustentabilidade. Através da criação de um copo feito a partir de um material recondicionado, dois problemas majoritários serão abrandados: a quantidade de lixo plástico produzido e a quantidade de rejeitos da produção do arroz, que, são essencialmente a palha e a casca, representando, respectivamente, 0,38 toneladas para cada tonelada de arroz colhido e $22 \%$ do peso total do grão. 


\section{RESULTADOS ESPERADOS}

Devido a qualidade dos projetos, alguns foram contemplados com bolsa de empreendedorismo (BIE) ofertado pela Secretaria de Desenvolvimento Tecnológico (SEDETEC) da UFRGS e ainda outros estão buscando auxílio junto ao fundo centenário da Escola de Engenharia tem como objetivo auxiliar as universidades de diversas maneiras, também conhecido como endowment funds ou fundos patrimoniais ou filantrópicos que são recursos advindos de doações de pessoas físicas e/ou pessoas jurídicas.

Ainda, tem-se a possibilidade de bolsas junto às agências de fomento como conselho nacional de desenvolvimento científico e tecnológico - CNPq e Fundação de Amparo à Pesquisa no Estado do Rio Grande do Sul - FAPERGS e parcerias externas.

Através dessa iniciativa de possibilitar ao acadêmico de ser inserido em pesquisas, apresentação de PITCH, elaborar artigos, participação de editais é uma forma de fazer com que aumente o interesse na engenharia e ainda em empreender, inovar, ser ousado e estar atento às mudanças tecnológicas e das novas exigências que elas acarretam fortalecer o intercâmbio de conhecimento entre alunos veteranos e calouros, pois, os veteranos, mestrandos e doutorandos são os mentores dos projetos.

\section{REFERÊNCIAS}

BESSANT, John; TIDD, Joe. 2009. Inovação e Empreendedorismo. Disponível em: https://books.google.com.br/books?hl=pt-BR\&lr=\&id=mV6kDwAAQBAJ\&oi=fnd\&pg=PR1 \&dq=inova $\% \mathrm{C} 3 \% \mathrm{~A} 7 \% \mathrm{C} 3 \% \mathrm{~A} 30+\mathrm{e}+$ empreendedorismo\&ots=NI9vhuTNbD\&sig=ayw5RIA2 vJU2sPdvW65iGdDd7cc\#v=onepage\&q=inova\%C3\%A7\%C3\%A30\%20e\%20empreended ori smo\&f=false. Acesso em: 27 maio. 2020

CONFEDERAÇÃO NACIONAL DA INDÚSTRIA. Fortalecimento das Engenharias. 2015. Confederação Nacional da Indústria. Disponível em: <http://arquivos.portaldaindustria.com.br/app/conteudo 24/2015/08/31/550/fortalecimento das engenharias web2.pdf>. Acessado em: 28 maio. 2020.

CORDEIRO, J. S. et al. Um futuro para a educação em engenharia no brasil: desafios e oportunidades. Revista de Ensino de Engenharia, v. 27, n. 3, p. 69-82, 2008.

CORRÊA, D. A.; NETO, M. S.; SPERS, V. R. E.; GIULIANI, A. C. Inovação, sustentabilidade e responsabilidade social: análise da experiência de uma empresa de equipamentos pesados. Dalila Alves Corrêa, Mário Sacomano Neto, Valéria Rueda Elias Spers, Antonio Carlos Giuliani Revista de Gestão Social e Ambiental 4 (3), 90-105, 2010.

ENGETELES, 2019. 4 Ferramentas para Análise de Falhas.Disponível em:

https://engeteles.com.br/ferramentas-para-analise-de-falhas/. Acesso em: 30 de maio. 2020.

LOUREIRO, S. M.; PEREIRA, V. L. D. V.; JÚNIOR, W. P. A sustentabilidade e o desenvolvimento sustentável na educação em engenharia. Revista Eletrônica em Gestão, Educação e Tecnologia Ambiental. Santa Maria, v.20, n.1, p. 306-324, 2016.

MARCO ANTÔNIO P. T.; ANA CRISTINA G. D.; SHANA H. W.; ADRIANO M. O. Adaptação à universidade em jovens calouros. Revista Semestral da Associação Brasileira de 
Psicologia Escolar e Educacional (ABRAPEE), São Paulo, Volume 12, 2008, Número 1, 185-202, janeiro/junho 2008.

PEREIRA. A. C.; SILVA G. Z.; CARBONARI M. E. E. Sustentabilidade, Responsabilidade Social e Meio Ambiente. Saraiva Educação S.A, 6 de outubro de 2017.

SERVIÇO BRASILEIRO DE APOIO ÀS MICRO E PEQUENAS EMPRESAS, 2020. Empreendedorismo e inovação: a moeda para o sucesso nos negócios. Disponível em: https://blog.sebrae-sc.com.br/empreendedorismo-e-inovacao/. Acesso em: 27 maio. 2020.

SERVIÇO BRASILEIRO DE APOIO ÀS MICRO E PEQUENAS EMPRESAS, 2019. Mas afinal, o que é empreendedorismo?Disponível em: https://blog.sebrae-sc.com.br/o-quee-empreendedorismo/. Acesso em: 27 maio. 2020.

SIMON, F, O.; BARROS FILHO, J.; SILVA, D.; SÀNCHEZ, C. G., 2002. Algumas tendências sobre habilidades e competências exigidas nos cursos de graduação em engenharia. In: CONGRESSO BRASILEIRO DE ENSINO DE ENGENHARIA, 30., 2002, Piracicaba. Anais eletrônico. Piracicaba: ABENGE, 2002, [CD-ROM].

SIMON, Fernanda Oliveira de. Habilidades e Competências em Engenharia: Criação e Validação de um Instrumento. 2004. Disponível em: http://repositorio.unicamp.br/bitstream/REPOSIP/253591/1/Simon FernandaOliveira M.pd

f Acesso em: 28 de maio de 2020.

\title{
THE NUCLEUS OF STUDENT ACTIONS AT FEDERAL UNIVERSITY OF RIO GRANDE DO SUL ENGINEERING SCHOOL: PROJECT OF RECEPTION OF THE FRESHMAN FROM ENGINEERING SCHOOL
}

\begin{abstract}
The Nucleus of Student Actions of the School of Engineering - NADI / EE of the Federal University of Rio Grande do Sul (UFRGS) has the purpose of supporting students in the development of their activities. Therefore, seeking to assist them within the proposed competencies, permanence, integration, and participation in the academic universe. Nevertheless, they feel that they are a fundamental part of the University, in a context of active participation during their academic trajectory. Thus, the project EE Freshmen Reception - Challenge "How to make UFRGS more sustainable" aims to enable the freshmen of the 13 EE courses, to identify gaps about the problems encountered in the experience of the University, for example, management of solid waste, living spaces, revitalization of areas, renewable energies In the edition of 2020/1, 7 (seven) projects were presented, of which 4 (four) are highlights, among them: Reliqua Biogás, Naturae Plastic, GURI: routes CONSERTA UFRGS, and, from this project, it is possible to develop experiential experiences, in the freshman, that allow the construction of diverse knowledge, which foster innovation, creativity and the entrepreneurial spirit of the students of different Engineering from UFRGS. Likewise, to stimulate the debate about the steps to undertake a business, analyze the influencing elements in this process and discuss issues of market
\end{abstract}


positioning, for example, check the initial investment to start the project and their return (payback).

Keywords: Engineering, Sustainability, Innovation, Challenge. 